\title{
Investigation of the Influence of Film Thickness on Phase Behavior of an Immiscible Binary Polymer Mixture
}

\author{
Manik Chandra Biswas* \\ Department of Textile Engineering, Chemistry and Science, North Carolina State University, USA
}

*Corresponding author: Manik Chandra Biswas, Department of Textile Engineering, Chemistry and Science, North Carolina State University, NC, USA.

Received Date: November 09, 2019

Published Date: November 15, 2019

\section{Abstract}

Recently, polymer phase behavior got much attention both in scientific research and industrial applications. Phase separation plays a vital role during practical applications of polymer mixture as the properties depend on its dynamics and structures. Researchers put intense interest to analyze phase separation of immiscible polymer blends and observed that various factors such as temperature, molecular weight, substrate and film thickness influence polymer phase separation process. Among them, film thickness dependence got immense attraction now a days. Film thickness dependence of phase separation of binary polymer mixture coupled with various analytical techniques, is discussed in Film thickness, phase separation, microscopic analysis, AFM, SEM, XPS, TEM this review.

Keywords: Film thickness; Phase separation; Microscopic analysis; AFM; SEM; XPS; TEM

\section{Introduction}

Analysis of polymer phase separation of blend system done extensively due to various industries such as microelectronics, coating, lubricant, wetting, diffusion and so on got interest in this field $[1,2]$. It is reported in the literature that the surface morphology of polymer blend is significantly different that in the bulk. This can be ascribed due to surface free energy difference of the polymers in the blend. Lower surface free energy component wants to enrich surface region to compensate the air-polymer interfacial free energy. In addition, the molecular accumulation near interfacial structure in thin films is greatly different compare to thick film as the polymeric chains are unstable in the interface [3]. Phase separation morphology for immiscible polymer blend films has been studied vastly by many researchers in the last decades [4-6].

Due to the advancement of nanotechnology, properties of nano templates and nanostructured polymeric parts depends vastly of ordered morphology of block copolymers in nanoscale domain.

In contrast, bulk behavior is easy to understand via phase diagram as a dependence of temperature. It can be also anticipated by total number of chains segment $(\mathrm{N})$, polymer composition (f) and Flory-Huggins interaction parameter $(\chi)$. The phase behavior found different in bulk compare to nanodomains [7].
Polymer blends dissolved in a common solvent and used to spin coat at room temperature to fabricate thin and ultrathin film preparation. The film thickness can be tuned via controlling solution concentrations and spin rates. The micron think film can prepare via solvent cast method. There is various substrate used in traditional polymer phase behavior analysis such as silicon wafer, gold, siliconized cover glass which showed different interaction with polymer systems [3]. Silicon wafer, which is hydrophilic in nature, substrate widely used due to availability, flexibility, cheap, easy to functionalize and so on compare to gold. The spin coated blend system, the air-polymer interface will be covered by both the polymer rich phases but for thick film, there will be lower surface free energy polymer overlayer. The surface covered with lower surface free energy polymer, is more stable due to no energy penalty. In case of spin coated sample, the solvent evaporates very fast before equilibrium stablished, which can cause chain conformation or accumulation structure because of freezing in thin film. Therefore, the phase behavior of thin films differs with that of thick films.

The following schematic shows the phase separation of PS/ PMMA blend on gold substrate [3] (Figure 1). 

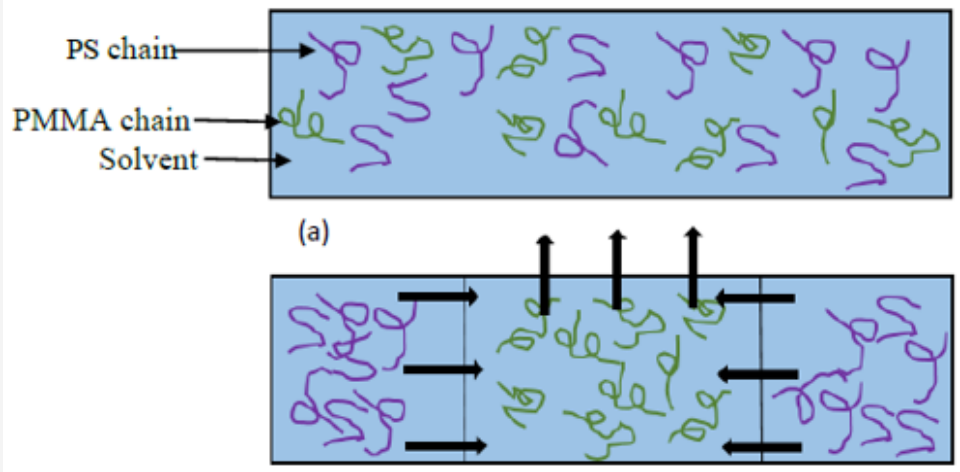

(b)

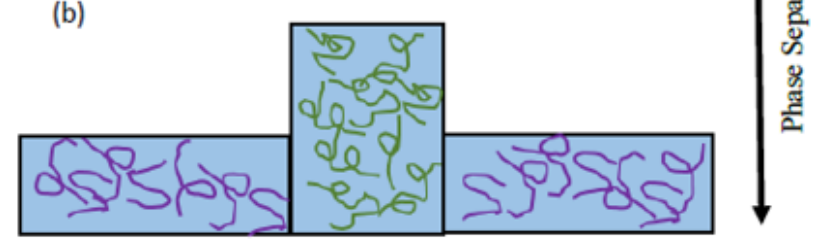

(c)

Figure 1: Schematic representation of the formation process of the surface phase-separated structure of the PS/PMMA thin film.

(a) The top diagram shows PS and PMMA segments in a solution.

(b) The center diagram corresponds to formation of the phase-separated structure on the way.

(c) The bottom picture shows a completed phase-separated morphology [3].

\section{Surface Morphology Characterization}

Most vastly used surface analysis techniques involve Atomic force microscopic (AFM) or field emission scanning electron microscopic (FE-SEM), X-ray photoelectron spectroscopic (XPS) and transmission electron microscopic (TEM) [3,8-10]. AFM analysis carried out to investigate the surface roughness of the films after phase separation. Confocal laser fluorescence microscopic analysis can also use to investigate depth profile of phase separated surface structure of blend system. To quantitative analysis of surface chemistry, XPS analysis $[10,11]$ can use at room temperature.

Some researchers also used grazing incidence small angle X-ray scattering (GISAXS), grazing incidence ultra-small-angle X-ray scattering (GIUSAX), and transmission electron microtomography (TEMT) analysis to investigate the surface morphology $[7,11]$. The following figure shows the spatial resolution of various surface analysis techniques [12] (Figure 2).

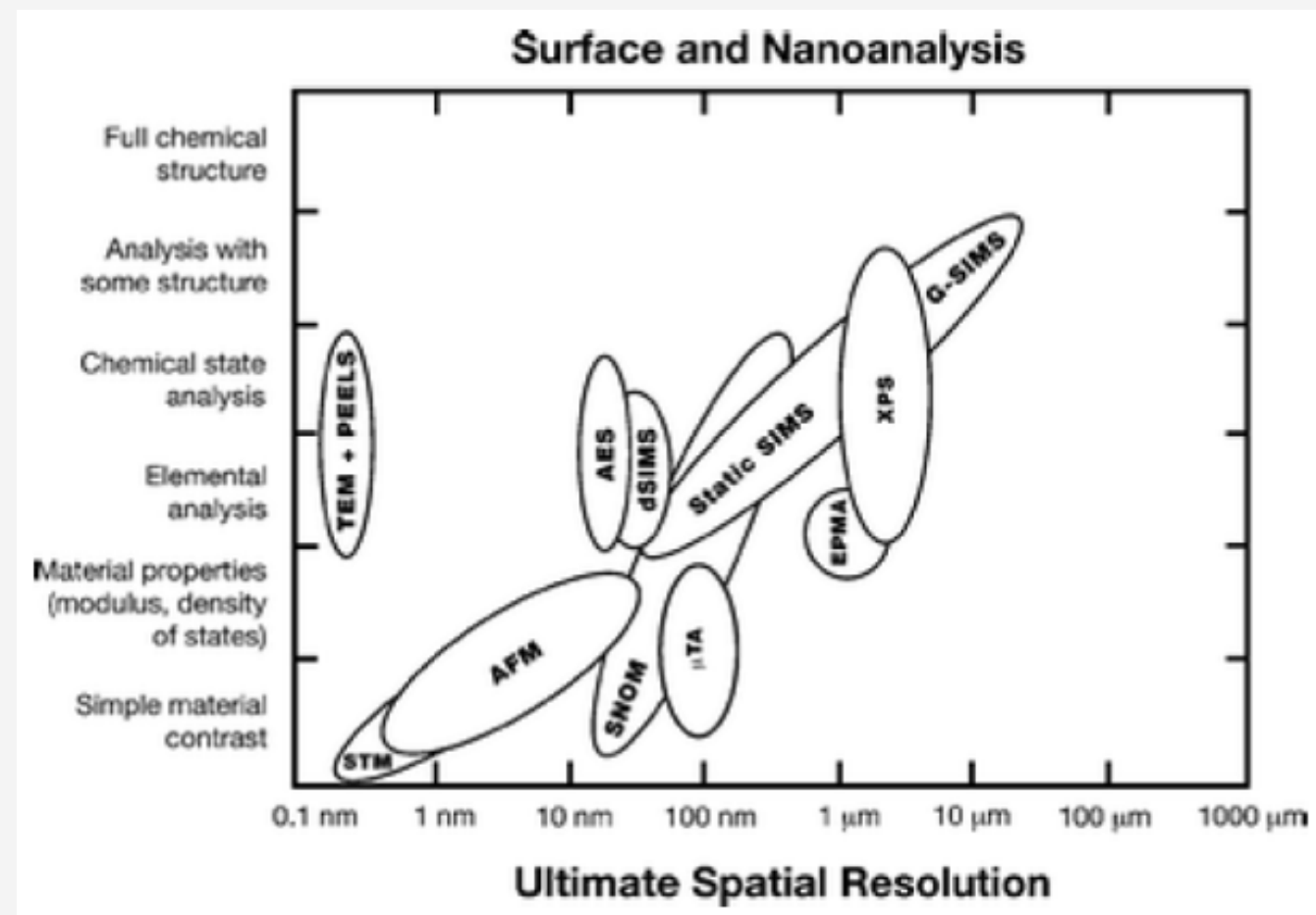

Figure 2: Comparison of common surface analysis techniques showing their relative spatial resolution and chemical information contents. 


\section{Analytical Techniques}

\section{AFM analysis}

The AFM is one kind of scanning probe microscopes (SPM). SPMs are designed to measure local properties, such as height, friction, magnetism, with a probe. To acquire an image, the probe scans over a small area of the sample, measuring the local property simultaneously. The surface morphology of the blend films can be investigated on the basis of AFM observation at room temperature. AFM cantilever used might be microfabricated and observation carried out in a repulsive manner. AFM allows to obtain very large magnifications up to $10^{8}$ and resolution of $0.1 \mathrm{~nm}$ in $\mathrm{X}, \mathrm{Y}$ and 0.01 in $\mathrm{Z}$ direction. It exhibits few advantages over SEM during imaging of dielectric materials like it does not need any sputtering of conductive materials and provides surface roughness without further processing. The prime significance of AFM is focused on surface quality on nanoscale level.

\section{FE-SEM analysis}

SEM scans a focused electron beam over a surface to create an image. The electrons in the beam interact with the sample, producing various signals that can be used to obtain information about the surface topography and composition. But this instrument is expensive and require proper maintenance.

Energy dispersive X-ray (EDX) coupled with SEM also used for qualitative analysis of the polymer films. EDX provides chemical composition of the individual component in the blend.

\section{XPS analysis}

XPS is also known as Electron Spectroscopy for Chemical Analysis (ESCA). It is one of the most widely used surface analysis techniques of various materials. The peak shape and precise position indicate the chemical state for the element. XPS is a surface sensitive technique because only those electrons generated near the surface escape and are detected. The photoelectrons of interest have relatively low kinetic energy. Due to its surface sensitivity, XPS used to investigate surface chemistry as most of the signal generates from surface. The XPS measurement performed under conventional conditions with a mono-energetic $\mathrm{Al} / \mathrm{Mg} \mathrm{K} \alpha$ x-ray source at $8 \mathrm{kV}$ and $30 \mathrm{~mA}$. The main chamber of the XPS instrument was maintained at $10^{-6} \mathrm{~Pa}$. XPS exhibits surface analysis resolution ca. $5 \mathrm{~nm}$ with a lateral spatial resolution as low as $7.5 \mu \mathrm{m}$. All C1s peaks were calibrated to a binding energy of $285.0 \mathrm{eV}$ for neutral carbon, in order to correct these charging energy shifts (Figure 3).

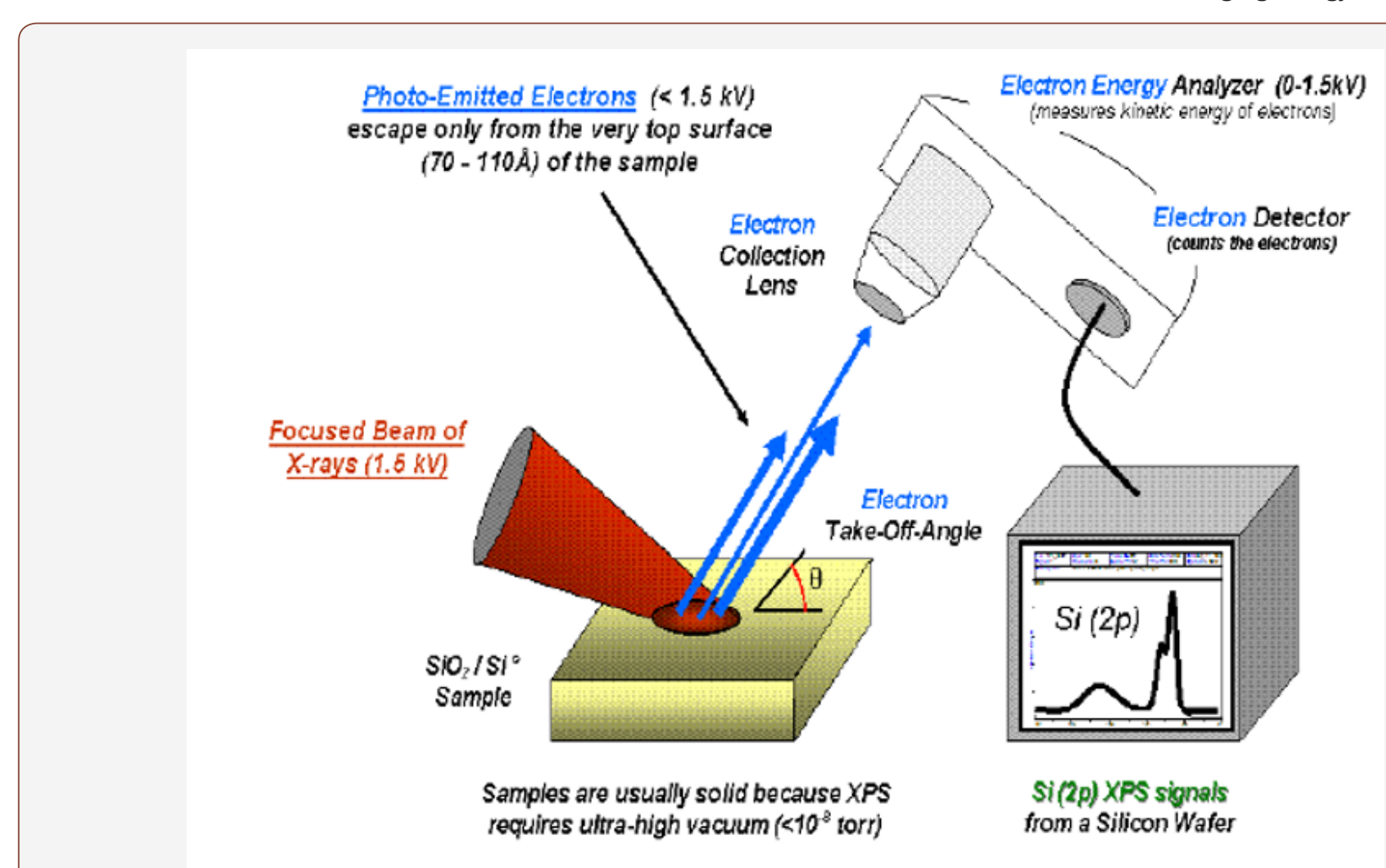

Figure 3: Schematic of classical XPS Analysis [13].

\section{Confocal laser fluorescence microscopic analysis}

This technique allows to identify various different molecules in the targeted area of the film. It helps to detect the specific molecules with the help of the fluorescence substances. The confocal fluorescence excites the sample via radiation and then detect the weaker emitted light from the sample as it has higher wavelength. It exhibits high resolution due to great control on depth of field, avoid image degradation and acquire series of images from thick samples.

\section{TEM analysis}

Transmission Electron Microscopy (TEM) provides powerful techniques for understanding various information of materials at very high spatial resolution, including morphology, size distribution, crystal structure, strain, defects, chemical information down to atomic level and so on. All the information that TEM can give to us are from electron-sample interaction. 
Bright Field images are formed by the direct-beam (transmitted beam) electrons, only very few scattered electrons can pass the aperture to contribute to the imaging. In Dark field TEM, an objective aperture is inserted into the back focal plane (where diffraction pattern is formed in the reciprocal space) to select scattered electrons. Typically, a specific diffracted beam (single crystalline) or a portion of a diffraction rings (polycrystalline) can be used for DF imaging. DF images can usually give information on nanocrystal size distribution and crystalline defects, such as stacking faults, twining, and dislocations.

\section{Conclusion and Outlook}

Due to lower surface free energy of polymer, the air-polymer interfacial region should be covered with polymer that specific polymer rich overlayer with a well-established macroscopic phase separated structure in the bulk with that of $2^{\text {nd }}$ polymer. The surface structure formation of the blend could be ascribed due to either chain conformation or accumulation structure being frozen at airpolymer interface because of fast solvent evaporation. It is also can be due to entanglements of polymer chains and interactions $(\chi)$.

\section{Acknowledgement}

This research did not receive any specific grant from funding agencies in the public, commercial, or not-for-profit sectors.

\section{Conflict of Interest}

The Author declares no conflict of interest.

\section{References}

1. Ma M, He Z, Yang J, Chen F, Wang K, et al. (2011) Effect of film thickness on morphological evolution in dewetting and crystallization of polystyrene/ poly ( $\varepsilon$-caprolactone) blend films. Langmuir 27(21): 13072-13081.

2. Liao Y, Jichun You, Tongfei Shi, Lijia An, Pradip Kumar Dutta (2007) Phase Behavior and dewetting for polymer blend films studied by in situ AFM and XPS: From thin to ultrathin films. Langmuir 23(22): 11107-11111.
3. Tanaka K, A Takahara, T Kajiyama (1996) Film thickness dependence of the surface structure of immiscible polystyrene/poly (methyl methacrylate) blends. Macromolecules 29(9): 3232-3239.

4. Scheffold F, Andrzej Budkowski, Ullrich Steiner, Erika Eiser, Jacob Klein (1996) Surface phase behavior in binary polymer mixtures. II. Surface enrichment from polyolefin blends. The Journal of chemical physics 104(21): 8795-8806.

5. Reich S, Y Cohen (1981) Phase separation of polymer blends in thin films. Journal of Polymer Science: Polymer Physics Edition 19(8): 12551267.

6. Newby BmZ, RJ Composto (2000) Influence of lateral confinement on phase separation in thin film polymer blends. Macromolecules 33(9): 3274-3282.

7. Jung J, Hae Woong Park, Sekyung Lee, Hyojoon Lee, Taihyun Chang, et al. (2010) Effect of film thickness on the phase behaviors of diblock copolymer thin film. ACS nano 4(6): 3109-3116.

8. Rangari VK, Vitus Apalangya, Manik Biswas, Shaik Jeelani (2017) Preparation and microscopic characterization of biobased nanoparticles from natural waste materials. Microscopy and Microanalysis 23(S1): 1938-1939.

9. Biswas MC, S Jeelani, V Rangari (2017) Influence of biobased silica/ carbon hybrid nanoparticles on thermal and mechanical properties of biodegradable polymer films. Composites Communications 4: 43-53.

10. Biswas MC, Boniface J Tiimob, Woubit Abdela, Shaik Jeelani, Vijaya K Rangari (2019) Nano silica-carbon-silver ternary hybrid induced antimicrobial composite films for food packaging application. Food Packaging and Shelf Life 19: 104-113.

11. You J, Liao Y, Men Y, Shi T, An L (2010) Film thickness dependence of phase separation and dewetting behaviors in PMMA/SAN blend films. Langmuir 26(18): 14530-14534.

12. Gilmore IS, Seah MP, Johnstone JE (2003) Quantification issues in ToF-SSIMS and AFM co-analysis in two-phase systems, exampled by a polymer blend. Surface and Interface Analysis: An International Journal devoted to the development and application of techniques for the analysis of surfaces, interfaces and thin films 35(11): 888-896.

13. (2019) X-ray photoelectron spectroscopy. Wikipedia. 